\title{
Ficus carica L. and Prunus spinosa L. extracts as new anthocyanin-based food colorants: A thorough study in confectionery products
}

\author{
Emanueli Backes $^{\mathrm{a}, 1}$, Maria G. Leichtweis ${ }^{\mathrm{a}, 1}$, Carla Pereira ${ }^{\mathrm{a}}$, Marcio Carocho ${ }^{\mathrm{a}}$, João C.M. Barreira ${ }^{\mathrm{a}}$, \\ Aziza Kamal Genena ${ }^{\mathrm{b}}$, Ilton José Baraldi ${ }^{\mathrm{b}}$, Maria Filomena Barreiro ${ }^{\mathrm{a}}$, Lillian Barros ${ }^{\mathrm{a}, *}$, \\ Isabel C.F.R. Ferreira, ${ }^{\text {a, }}$ \\ ${ }^{a}$ Centro de Investigação de Montanha (CIMO), Instituto Politécnico de Bragança, Campus de Santa Apolónia, 5300-253 Bragança, Portugal \\ ${ }^{\mathrm{b}}$ Departamento Acadêmico de Alimentos (DAALM), Universidade Tecnológica Federal do Paraná, Campus Medianeira, 85884-000 Paraná, Brazil
}

A R T I C L E I N F O

\section{Keywords:}

Ficus carica L. peel

Prunus spinosa L. epicarp

Natural colorants

Icing, pastry

\begin{abstract}
A B S T R A C T
The present work describes the evaluation of fig peels and blackthorn fruit extracts as natural purple colorants in doughnuts (icing) and in a typical Brazilian pastry called "beijinho". The extracts were screened for their antioxidant activity as well as their antibacterial capacity. Nutritionally, the employed extracts did not induce significant changes, contrarily to the observed for the rheological features, mainly the darker purple tone observed when blackthorn extract was used in the icing solution. After $24 \mathrm{~h}$, both prepared formulations showed a decrease in color intensity, with no significant differences between fig and blackthorn extracts. In turn, the firmness and consistency of the doughnuts benefited from using natural colorants in the icing solution, while "beijinhos" became softer and chewier, which are valued attributes. A significant increase in the antioxidant and antimicrobial activities was also observed for both natural extracts. Accordingly, the evaluated extracts are promising candidates as natural food colorants.
\end{abstract}

\section{Introduction}

Food colorants are substances of natural or synthetic origin used to convey, restore or standardize the color and appearance of food, making it more attractive to consumers (Gordillo et al., 2018). Due to consumer preference for natural food colorants, the quest for pigments from natural sources has become widespread, quickly turning into a trend in the current market (Agcam, Akyildiz, \& Balasubramaniam, 2017; Gordillo et al., 2018).

Anthocyanins represent the largest group of phenolic pigments and the most important group of water-soluble pigments in plants. These compounds are responsible for colors in fruits, vegetables, cereal grains and flowers (Mojica, Berhow, \& Gonzalez de Mejia, 2017), and, beyond this, they also display health benefits, namely in reducing the risk of coronary heart disease, stroke, and other diseases, while also showing cytotoxic and anti-inflammatory effects (Agcam et al., 2017). As watersoluble and innocuous pigments, anthocyanins have high potential as food colorants, besides adding value due to their bioactivities. Color formulations based on anthocyanins exhibit some limitations due to their lack of chemical stability. Factors such as $\mathrm{pH}$ variation, exposure to heat, light, oxygen, temperature, metals, bleaching agents, among others, can affect their behavior and stability, compromising the desired color. In light of these limitations, the incorporation of these compounds constitutes a challenge for the food industry (Gordillo et al., 2018).

The fig tree (Ficus carica L., family Moraceae) is native to southwestern Asia and the eastern part of the Mediterranean. Its fruit, is a rich source of sugar, vitamins and phenolic compounds (BuenrostroFigueroa et al., 2017; Duenas et al., 2008), being consumed fresh or dehydrated. Figs are also consumed as processed foods, mainly as ingredients for wine, liquor, juice and jelly, although these foods use the pulp and discard the peel, which is the largest reservoir of phenolic compounds (Buenrostro-Figueroa et al., 2017). In fact, Vallejo, Marín, and Tomás-Berberán (2012), showed that high concentrations of phenolic compounds (mainly anthocyanins) were found in the peel of mature figs (Barolo, Ruiz Mostacero, \& López, 2014).

Prunus spinosa L. has also proved its worth as a fruit with high amounts of antioxidant molecules and natural pigments. The fruits of this plant, also known as blackthorn, presents high amounts of cyanidin 3-O-rutinoside and peonidin 3-O-rutinoside, as well as an overall high

\footnotetext{
* Corresponding authors.

E-mail addresses: lillian@ipb.pt (L. Barros), iferreira@ipb.pt (I. C.F.R. Ferreira).

${ }^{1}$ These authors contributed equally to this article
} 
amount of antioxidant compounds (Guimarães et al., 2013, 2014; Barros, Carvalho, Morais, \& Ferreira, 2010). The fruits of $P$. spinosa are commercially unexplored due to their astringent taste, being basically used for the production of liqueurs and jams and also for some medicinal purposes (Morales et al., 2013; Pinacho et al., 2015).

The present study aims to evaluate the bioactive properties and applicability of natural food colorants based on anthocyanins from the residues (peels and epicarps) of Ficus carica L. and Prunus spinosa L. These extracts were tested on two highly appreciated deserts, namely doughnuts (as an icing solution) and a Brazilian soft dairy pastry called "beijinho", being their application monitored in terms of chemical and physical properties immediately after manufacture and after $24 \mathrm{~h}$, according to their typical shelf-life.

\section{Material and methods}

\subsection{Standards and reagents}

The reference standard mixture of fatty acid methyl esters (FAME) (47885-U standard), 2,2'-azobis (2-amidinopropane) dichlorohydrate (AAPH), acetic acid, formic acid, trichloroacetic acid) and Tris were purchased from Sigma-Aldrich, as well as the sugar standards used. Hydrogen chloride, formic acid, HPLC grade acetonitrile and P.A. grade ethanol were obtained from Fisher Scientific. Toluene, sulfuric acid, and the remainder of the chemical reagents used were purchased from Sigma Chemical Co. Water was treated in a Milli-Q purification system (TGI Pure Water Systems). Anthocyanin standards (cyanidin-3-O-rutinoside and cyanidin-3-O-glucoside) were purchased from Extrasynthèse.

\subsection{Preparation of the extracts}

The figs and blackthorns were obtained from a local producer in Bragança, Portugal ( $41^{\circ} 47.422, \mathrm{~W} 6^{\circ}$ 45.606). The samples were peeled, and the peels of $F$. carica and the epicarp of $P$. spinosa were lyophilized (FreeZone 4.5, Labconco), crushed to 20 mesh and stored in a freezer at $-20{ }^{\circ} \mathrm{C}$ for subsequent analysis. The lyophilized extracts were extracted under the best conditions according to a previous study (Backes et al., 2018; Leichtweis et al., 2019), relying on an ultrasonic probe apparatus (QSonica sonicators, model CL-334, Newtown, CT, USA), where each plant was extracted with $100 \mathrm{~mL}$ of acidified solvent ( $\mathrm{pH} 3$, using citric acid). For the fig peel extraction, the solvent used was 100\% ethanol (180 g/L, $21 \mathrm{~min}, 310 \mathrm{~W}$ ), whilst for blackthorn, the solvent used was a mixture $(50: 50 \mathrm{v} / \mathrm{v})$ of ethanol:water $(75 \mathrm{~g} / \mathrm{L}, 5 \mathrm{~min}$, $400 \mathrm{~W})$. The samples were centrifuged $\left(6000 \mathrm{rpm}\right.$ for $20 \mathrm{~min}$ at $\left.10{ }^{\circ} \mathrm{C}\right)$ and filtered through filter paper Whatman $n^{\circ} 4$ to remove suspended solids. The supernatants were lyophilized (FreeZone 4.5, Labconco) and stored in a refrigerator $\left(-20^{\circ} \mathrm{C}\right)$ for subsequent analyzes.

\subsection{Bioactivities}

\subsubsection{Evaluation of antioxidant activity: Inhibition of lipid peroxidation (TBARS)}

The antioxidant activity of the extracts was evaluated by the lipid peroxidation inhibition using thiobarbituric acid reactive substances (TBARS) in pig brain homogenates. Successive dilutions of the extract were prepared and mixed with ascorbic acid, brain tissue, and thiobarbituric acid. The potential formation of malodialdehyde (MDA) from brain tissue, which occurs in the absence of antioxidant agents, was verified by the formation of MDA-TBA complex (intense pink color) at $532 \mathrm{~nm}$ (Barros et al., 2010). A percentage inhibition versus concentration curve of the extract was created using the following equation:

Inhibition percent $(\backslash \%)=\left[\frac{\mathrm{CA}-\mathrm{EA}}{\mathrm{CA}}\right] \times 100$
CA represents the control absorbance and EA the extract solution absorbance. The concentration capable of causing 50\% inhibition of lipid peroxidation $\left(\mathrm{IC}_{50}\right)$ was determined for each of the extracts.

\subsubsection{Evaluation of antioxidant activity: Inhibition of oxidative hemolysis (OxHLIA)}

A known mass of extract was dissolved in a phosphate buffered saline solution, obtaining concentrations ranging from 312.5 to $5000 \mu \mathrm{g} / \mathrm{mL}$. To determine the inhibition capacity of the oxidative hemolysis (OxHLIA) associated with each of the extracts, blood from healthy sheep was harvested and used as a substrate. After filtering the blood, the leucocytes were discarded, and the red cell suspension was used after washing with a $\mathrm{NaCl}$ solution. The erythrocytes were resuspended and incubated at $37{ }^{\circ} \mathrm{C}$ for $10 \mathrm{~min}$, prior to an optical reading at $690 \mathrm{~nm}$ every $10 \mathrm{~min}$ for $2 \mathrm{~h}$. The percentage of the intact erythrocyte population (EP) was calculated using the following equation:

$\mathrm{EP}(\%)=\left(\mathrm{S}_{\mathrm{t}}-\mathrm{CH}_{0} / \mathrm{S}_{0}-\mathrm{CH}_{0}\right) \times 100(2)$

$S_{t}$ and $S_{0}$ correspond to the optical density of the sample at time $t$ and 0 min, respectively, and $\mathrm{CH}_{0}$ is the optical density of complete hemolysis at $0 \mathrm{~min}$. The results were expressed as hemolysis delay time $(\Delta \mathrm{t})$. Subsequently, linear correlations were established between the $\Delta \mathrm{t}$ values and the different sample concentrations (Takebayashi et al., 2012). The extract concentration capable of delaying $80 \%$ of the hemolysis $\left(\mathrm{IC}_{80}\right)$ in $60 \mathrm{~min}\left(\mathrm{IC}_{80(60 \mathrm{~min})}, \mathrm{mg} / \mathrm{mL}\right)$ and $120 \mathrm{~min}\left(\mathrm{IC}_{80(120}\right.$ $\mathrm{min}), \mathrm{mg} / \mathrm{mL}$ ) were calculated.

\subsubsection{Evaluation of antibacterial activity}

The assay used microorganisms obtained from clinical isolates (Hospital Centre from Trás-os-Montes and Alto Douro, Vila Real, Portugal). Four Gram-positive bacteria (Enterococcus faecalis, isolated from urine; Listeria monocytogenes, isolated from cerebrospinal fluid; methicillin-resistant Staphylococcus aureus - MRSA, isolated from expectoration and methicillin-sensitive Staphylococcus aureus - MSSA, isolated from wound exudate) and five Gram-negative bacteria (Pseudomonas aeruginosa and Proteus mirabilis, isolated from expectoration; Escherichia coli, Klebsiella pneumoniae and Morganella morganii, isolated from human urine).

Microorganism identification and susceptibility tests were performed on the MicroScan panels (MicroScan ${ }^{\circledR}$; Siemens Medical Solutions Diagnostics, West Sacramento, CA, USA) using the microdilution method. The interpretation criteria was based on Interpretive Breakpoints as indicated in the Clinical and Laboratory Standards Institute (CLSI, 2008) and in the European Committee on Antimicrobial Susceptibility Testing (EUCAST, 2013).

The colorimetric assay was performed using $p$-iodonitrotetrazolic chloride (INT) according to Kuete et al. (2011) with some modifications. Successive extract dilutions were made in microplate wells (20 to $0.156 \mathrm{mg} / \mathrm{mL}$ ) prior to inoculation with a suitable turbidity suspension. Three negative controls (MHB/TSB; MHB/TSB with bacterial inoculum and antibiotic; extract) and a positive control (MHB/TSB with bacterial inoculum) were also prepared. For the Gram-positive bacteria, Vancomycin was used as a positive control, while Ampicillin and Imipenem were used for the Gram-negative ones. The minimum inhibition concentration (MIC) was defined as the lowest extract concentration to inhibit bacterial growth (color change from yellow to pink), while the minimum bactericidal concentration (MBC) was the minimum concentration that killed all bacteria present in the well.

\subsubsection{Evaluation of cytotoxic potential in a non-tumor cell line}

Pig liver obtained from a local slaughterhouse was used to obtain a non-tumor cell culture, henceforth named PLP2 (primary culture of pig liver cells). The liver tissue was washed with Hank's solution and divided into explants of approximately $1 \times 1 \mathrm{~mm}^{2}$, obtaining a cell culture with adequate density $\left(190 \mu \mathrm{L}, 1.0 \times 10^{4}\right.$ cell/well), which was pipetted in 96-well microplates with $10 \mu \mathrm{L}$ of different dilutions of the extracts (400 to $1.5 \mu \mathrm{g} / \mathrm{mL}$ ) and incubated at $37{ }^{\circ} \mathrm{C}$ for $48 \mathrm{~h}$. Analysis of 
the cytotoxicity of the extract was evaluated according to the Sulforhodamine B (SRB) colorimetric assay. Ellipticine was used as a positive control and the results were expressed as $\mathrm{GI}_{50}$.

\subsection{Incorporation of extracts into foodstuffs}

After confirming the antioxidant and antibacterial activity of fig peel and blackthorn extracts, as well as their lack of toxicity, the extracts were incorporated in the doughnut icing and "beijinhos"

\subsubsection{Preparation of doughnut icing}

White chocolate was melted in a heated bath and homogenized with milk cream at a $3: 1 \mathrm{~m} / \mathrm{m}$ (840 g of white chocolate to $280 \mathrm{~g}$ of milk cream) ratio. Then, citric acid was added at $1: 200 \mathrm{~m} / \mathrm{m}(4.2 \mathrm{~g})$ for chocolate (below the maximum value of $5 \mathrm{~g} / \mathrm{kg}$ stipulated by legislation (European Parliament, 2011). The mixture was then divided into three portions, in one portion, acting as a dye, the extract of fig peel was added, in the second portion, the extract of blackthorn was added (both at $0.7 \%$ of the total volume of the icing), and finally, in portion three, no colorant was added. Each of these portions was further divided into two three replicates for each analysis, at $0 \mathrm{~h}$ and $24 \mathrm{~h}$ measuring both physical, chemical and nutritional parameters.

\subsubsection{Preparation of "beijinho"}

In the preparation of the "beijinho" $800 \mathrm{~g}$ of condensed milk, $200 \mathrm{~g}$ of milk cream and $24 \mathrm{~g}$ of butter were used. These ingredients were heated and stirred until the mixture was easily detached from the bottom of the pan. Then, $4.8 \mathrm{~g}$ of citric acid was added, and the mixture separated in three equal parts. One of them was used as control, and the other two were incorporated with $2.4 \mathrm{~g}$ one of the two natural extracts. After cooling, the dough was divided into $10 \mathrm{~g}$ portions and rolled into small spheres (typical presentation of this pastry).

\subsection{Evaluation of the nutritional profile}

The nutritional composition of the doughnut icing and "beijinhos" samples was performed by analyzing the moisture content, ashes, proteins, fat and carbohydrates according to the procedures described by AOAC (2016). Moisture was determined by lyophilization (FreeZone 4.5, Labconco) following AOAC 925.09 method. The ashes were determined by muffle incineration (Optic ivymen system) at $550 \pm 5{ }^{\circ} \mathrm{C}$ following AOAC 923.05. The total proteins were estimated by the macro-Kjeldahl technique in a specific equipment (Pro-Nitro A, Selecta) by determining the total nitrogen present in the sample $(\mathrm{N} \times 6.25$ for the icing and $\mathrm{N} \times 6.38$ for "beijinho") following method AOAC 920.87 . Total fat was determined by extraction through a Soxhlet apparatus using method AOAC 989.05. The carbohydrate content ( $\mathrm{g} / 100 \mathrm{~g} \mathrm{fw})$, in fresh product (fw), was calculated by difference and the total energy (kcal/100 g fw) was determined according to the following equation.

Energy $=4 \times(\mathrm{CH}+\mathrm{PC})+9 \times \mathrm{CF}(3)$

$\mathrm{CH}$ represents carbohydrates $(\mathrm{g} / 100 \mathrm{~g} \mathrm{fw})$, PC the protein content $(\mathrm{g} / 100 \mathrm{~g} \mathrm{fw})$, CF the crude fat $(\mathrm{g} / 100 \mathrm{~g} \mathrm{fw})$.

\subsection{Individual molecules}

Free sugars and individual fatty acids were also determined, namely through gas chromatography coupled to flame ionization detector (GCFID) and high-performance liquid chromatography coupled to refraction index detector (HPLC). The free sugars followed the methodology published by Barros et al. (2013), where they were analysed by HPLC coupled to a refraction index detector (Knauer, Smartline system 1000). The compounds were identified by chromatographic comparisons with authentic standards (D(-)-fructose, $\mathrm{D}(+)$-sucrose, $\mathrm{D}(+)$-glucose, and $\mathrm{D}$ (+)-lactose, as also melezitose which was applied as the internal standard (IS) and used in the quantification method. Data was analyzed using Clarity 2.4 software (DataApex, Podohradska, Czech Republic), and the results were expressed in $\mathrm{g} / 100 \mathrm{~g}$ fw.

Fatty acids were determined by GC-FID (DANI model GC 1000, Contone, Switzerland). The separation was achieved with a MachereyNagel (Düren, Germany) column (50\% cyanopropyl-methyl-50\% phenylmethylpolysiloxane, $30 \mathrm{~m} \times 0.32 \mathrm{~mm}$ i.d. $\times 0.25 \mu \mathrm{m} \mathrm{d}_{\mathrm{f}}$ ). Fatty acids were identified by comparing their retention times to the ones of FAME peaks of commercial standards (FAME reference standard mixture, standard 47885-U, Sigma-Aldrich, St. Louis, MO, USA). The results were recorded and processed using the CSW 1.7 software (DataApex 1.7, Prague, Czech Republic) and were expressed in relative percentage (\%).

\subsection{Evaluation of $\mathrm{pH}$, color and texture parameters}

The $\mathrm{pH}$ was measured directly in three different points of the samples using a portable pH-meter (Hanna Instruments, Woonsocket, Rhode Island, USA). The external color was measured in three different points of the three replicates using a portable colorimeter (model CR400, Konica Minolta Sensing Inc., Tokyo, Japan) relying on the illuminant $\mathrm{C}$ with a diaphragm aperture of $8 \mathrm{~mm}$, as well as the CIElab $L^{*}$ (luminosity), $a^{*}$ (green/red) and $b^{*}$ (blue/yellow) color space values. The total color difference $\left(\Delta \mathrm{E}^{*}\right)$ between the colored samples and the control sample was also calculated using the following equation:

$\Delta \mathrm{E}^{*}=\sqrt{\left(L_{2}^{*}+L_{1}^{*}\right)^{2}+\left(a_{2}^{*}+a_{1}^{*}\right)^{2}+\left(b_{2}^{*}+b_{1}^{*}\right)^{2}}$

Texture was determined using a TA XT. Plus Texture Analyzer (Stable Micro System) to measure the following parameters: firmness, consistency, cohesiveness and the viscosity index of the icing, using an inverted extrusion probe, due to be a semiliquid. The "beijinhos", being a solid, underwent a normal TPA (texture profile analysis) analysis, describing the hardness, adhesiveness, resilience, cohesiveness, springiness, gumminess and chewiness, using an acrylic disc $(40 \mathrm{~mm})$, a $30 \mathrm{~kg}$ load cell, speeds of $3 \mathrm{~mm} / \mathrm{s}$ and $25 \%$ strain with a trigger force of $50 \mathrm{~g}$.

\subsection{Statistical analysis}

All samples are expressed as mean $\pm \mathrm{SD}$, using 3 replicates for all assays. In relation to the incorporation of the extracts, samples were analyzed through a two-way analysis of variance (ANOVA) with type III sums of squares, after verification of the homoscedasticity through a Levene's test. The Tukey (homoscedastic) or Tamhane's T2 (heteroscedastic) post-hoc tests were used to classify differences induced by the colorant type (CT), while a student-T test was used with the same purpose for the analysis time (AT). Using a two-way ANOVA, the contribution of each CT or AT can be assessed individually, and their influence in the variation of each parameter can be better understood. When a significant interaction $(p<0.05)$ among the two factors $(\mathrm{CT} \times \mathrm{AT})$ was found, they are evaluated simultaneously and, in some cases, general tendencies are extracted from the Estimated Marginal Means (EMM) plots. If not, they were classified using the post-hoc tests described above. All statistical analysis was carried out using the IBM SPSS, version 25.0. (IBM Corp.).

\section{Results and discussion}

\subsection{Antioxidant activity}

The antioxidant potential of the fig peel extract, rich in cyanidin 3rutinoside (Backes et al., 2018), and blackthorn extract, rich in cyanidin 3-rutinoside and peonidin 3-rutinoside (Leichtweis et al., 2019), were evaluated by two distinct methods, which involve the use of biological material, namely the TBARS and OxHLIA assays. In what concerns the OxHLIA method, for the fig extract, the results obtained did not allow the establishment of $\mathrm{EC}_{50}$ values, because the different extract 


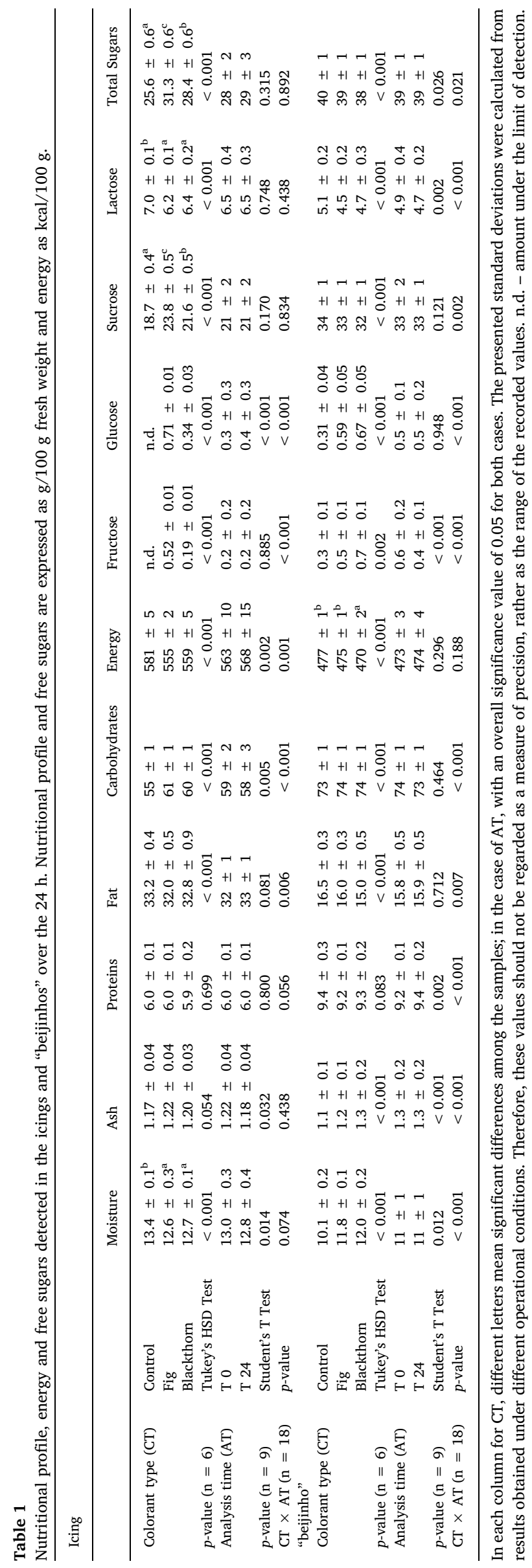

concentrations presented different antioxidant behaviors. These effects are shown in Fig. S1 (supplementary material), where it is possible to observe the overlap and/or crossing of the lines corresponding to the different hemolytic times (Ht) of each concentration. Similar results were found in previous studies, leading to the same type of conclusions (Takebayashi et al., 2012). The $\mathrm{IC}_{80}$ value, corresponding to the concentration capable of delaying hemolysis was determined at 60 (IC 80 (60 min) $)$ and in $120 \mathrm{~min}\left(\mathrm{IC}_{80}(120 \mathrm{~min})\right.$ ), which are also shown in Table S1 (supplementary material). For the blackthorn extract the $\mathrm{IC}_{50}$ was calculated, although for comparison sake between the two extracts, only the $\mathrm{IC}_{80}$ are reported. None of the extracts showed IC $\mathrm{I}_{50}$ and $\mathrm{IC}_{80}$ values as low as Trolox (used as positive control), although it should be noted that this compound is analyzed in its pure form. Whole extracts, despite potential synergies among different compounds, are not expected to be as antioxidant as pure compounds (owing the purity degree). Overall, blackthorn presented greater cell protection against oxidation when compared to the fig peel extract. These results are in agreement with those found in other studies, which had already reported high antioxidant activity for both extracts (Amessis-Ouchemoukh et al., 2017; Barros et al., 2010; Pereira et al., 2017; Pinacho et al., 2015; Solomon et al., 2006). A considerable number of studies have shown the antioxidant activity of $F$. carica peel, namely Harzallah et al. (2016) which determined the $\mathrm{IC}_{50}$ values of 9.71 and $4.52 \mathrm{mg} / \mathrm{mL}$ for the purple and black variety peel, respectively, using the (2,2-diphenyl-1-picrylhydrazil) DPPH free radical method. Oliveira et al. (2009) obtained $\mathrm{IC}_{25}$ values of $2.95 \mathrm{mg} / \mathrm{mL}$ for the "Pingo de Mel" variety of figs, while the pulp of the same did not present a decent DPPH capacity, even at a concentration of $5.56 \mathrm{mg} / \mathrm{mL}$.

Concerning blackthorn, Barros et al. (2010) reported an IC $\mathrm{C}_{50}$ value of $0.154 \pm 0.002 \mathrm{mg} / \mathrm{mL}$ for methanolic extracts of whole fruits using the TBARS assay. When compared, these results corroborate the antioxidant potential of the epicarp extract of $P$. spinosa. The small difference between them is justified by the different amount and/or phenolic profile present in the analyzed extracts, due to factors like solvent type, extraction conditions, part of the fruit used and conditions of soil and climate of the year of harvest, along with variety and individual variation. The $\mathrm{IC}_{50}$ values determined in this study for the TBARS method regarding fig and blackthorn are higher than those determined in other studies in natural matrices already commercially reported as rich sources of antioxidant compounds such as blackberry (Aristotelia chilensis) with an $\mathrm{IC}_{50}$ of $0.024 \mathrm{mg} / \mathrm{mL}$ (Céspedes et al., 2008) and olive oil (Olea europaea) with an $\mathrm{IC}_{50}$ of $44.71 \mu \mathrm{g} / \mathrm{mL}$ (Khaliq et al., 2015). However, compared to other alternative sources used to recover phenolic compounds, both extracts investigated in the present study had superior antioxidant activity.

\subsection{Antibacterial activity}

The samples were tested against a set of 9 Gram-positive and Gramnegative bacteria strains with importance in the health area, some of them with known resistance to commercial antibiotics. All bacteria were somehow inhibited, making it possible to obtain minimum inhibition concentrations (MIC) values. For both extracts, there were no specific groups of bacteria that presented greater sensitivity or resistance for both extracts, since the results were heterogeneous. This suggests that the samples have compounds with an ample range of activity, being able to act against Gram-positive and Gram-negative bacteria. For the fig extract, the best MIC result (the lowest concentration required to inhibit the growth of the microorganism), was detected in one Gram negative (Escherichia coli) and in two Gram positive (MRSA and MSSA) species, all with a MIC of $2.5 \mathrm{mg} / \mathrm{mL}$. Regarding blackthorn, the lowest result was also $2.5 \mathrm{mg} / \mathrm{mL}$, observed in MSSA. In general, MIC values obtained for fig extract were all lower or equal to that obtained for blackthorn, which demonstrates a slightly higher antibacterial activity than the one obtained for fig peel. Pertuzatti et al. (2016) evaluated the antimicrobial activity of ten 

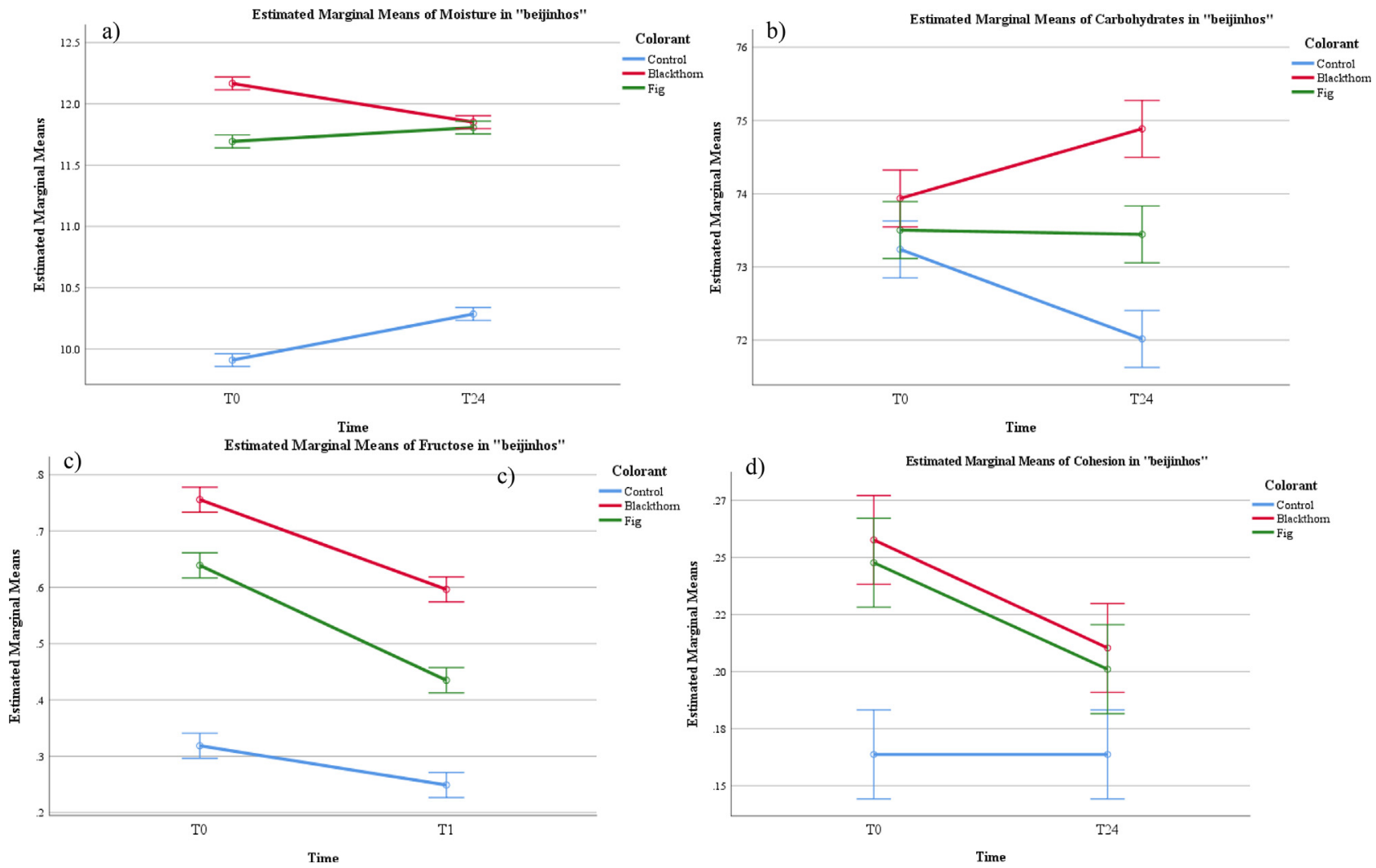

Fig. 1. EMM plots of "beijinhos" for a) Moisture, b) carbohydrates, c) fructose and d) cohesiveness.

blueberry cultivars and verified higher MIC's $(20 \mathrm{mg} / \mathrm{mL})$ than those obtained in the present study for the fruit epicarp extract of $P$. spinosa. In a study by Liu et al. (2017) with grapefruit peels, the MIC obtained for $E$. coli was $4.5 \mathrm{mg} / \mathrm{mL}$, presenting an antimicrobial activity slightly higher to the one of blackthorn, but inferior to fig extract. These two extracts presented better antimicrobial activity than that reported in the study by Diao et al. (2013), who determined a MIC value of $10 \mathrm{mg} / \mathrm{mL}$ for $E$. coli and $20 \mathrm{mg} / \mathrm{mL}$ for $P$. aeruginosa using the essential oil extracted from Zanthoxylum schinifolium, a common edible plant from Asia. Still, and comparatively to E. coli, Yao et al. (2011) determined a MIC of $8.28 \mathrm{mg} / \mathrm{mL}$ of the Bayberry fruit (Myryca gale), widely consumed in China, which also had MIC higher than that of both extracts studied herein.

\subsection{Cytotoxic potential}

From the performed analysis, both extracts showed no activity against the non-tumor porcine liver cell line PLP2, revealing no toxicity to these cells, even at a maximum concentration of $400 \mu \mathrm{g} / \mathrm{mL}$. This is important to make a first validation of the use and incorporation of these extracts in food matrices. The same conclusion was reached by (Uz et al., 2015), who described fig extract (whole infructescence) as a natural matrix that did not present a cytotoxic effect on the investigated normal cell line (rat epithelial cell).

\subsection{Application of the colorant extracts in a food product}

The extracts were used as natural food colorants in two dairy confectionery products, doughnut icings and "beijinho" pastry, being analyzed immediately after production and after $24 \mathrm{~h}$ of shelf-life. This narrow window for analysis is due to the high perishability of these food products, which are made without any preservatives (used to keep the external color and avoid oxidation and microbial contamination).

\subsection{Nutritional profile and individual sugars}

The nutritional profile encompassed the analysis of moisture, ash, proteins, fat, carbohydrates and energy of all samples (colored with fig extract, colored with blackthorn and control sample: no colorant). As stated in section 2.8., a two-way ANOVA was used to obtain conclusions regarding the contribution of each individual factor: colorant type (CT) and analysis time (AT). In all cases showing independent effects for each parameter ( $p$-value CT $\times$ AT $>0.05$ ), each sample was classified individually; on the other hand, when CT $\times$ AT had a $p$-value $<0.05$, the overall conclusions were extracted from the plots of the EMM. Considering this, Table 1 is divided into 4 horizontal sections. The two upper ones pertain to the nutritional profile of the doughnut icing, and the two bottom ones to "beijinhos". The results for each confectionery product are further divided in two sections: CT (top) and AT (bottom). For each colorant type, both analysis times are included, and for each analysis time, all the colorants are included, justifying the apparently high standard deviation values (results obtained in different conditions). Still, this allows to understand the contribution of each factor independently. Considering the nutritional profile of the icings (Table 1), carbohydrates were the highest nutrient, followed by fat. For moisture the highest differences were probably due to the incorporation of the extracts. Beyond this, the incorporation of the extracts and the $24 \mathrm{~h}$ of storage had a very slight effect. For "beijinhos", a significant interaction was found for all nutrients except energy value, in which blackthorn showed lower values. Fig. 1 shows the EMM plots of "beijinhos", where it became clear that moisture (Fig. 1a) was higher for samples with natural colorants (when compared to the control), and these tended to increase their moisture after $24 \mathrm{~h}$, probably due to its absorption from the environment. Fig. $1 \mathrm{~b}$ shows a carbohydrates reduction in the control samples of "beijinhos" after $24 \mathrm{~h}$, while the same parameter increased in samples with blackthorn extract and showed no variation in samples with fig extract. Once again, these are general tendencies extracted from the EMM plots, and do not show a significant 


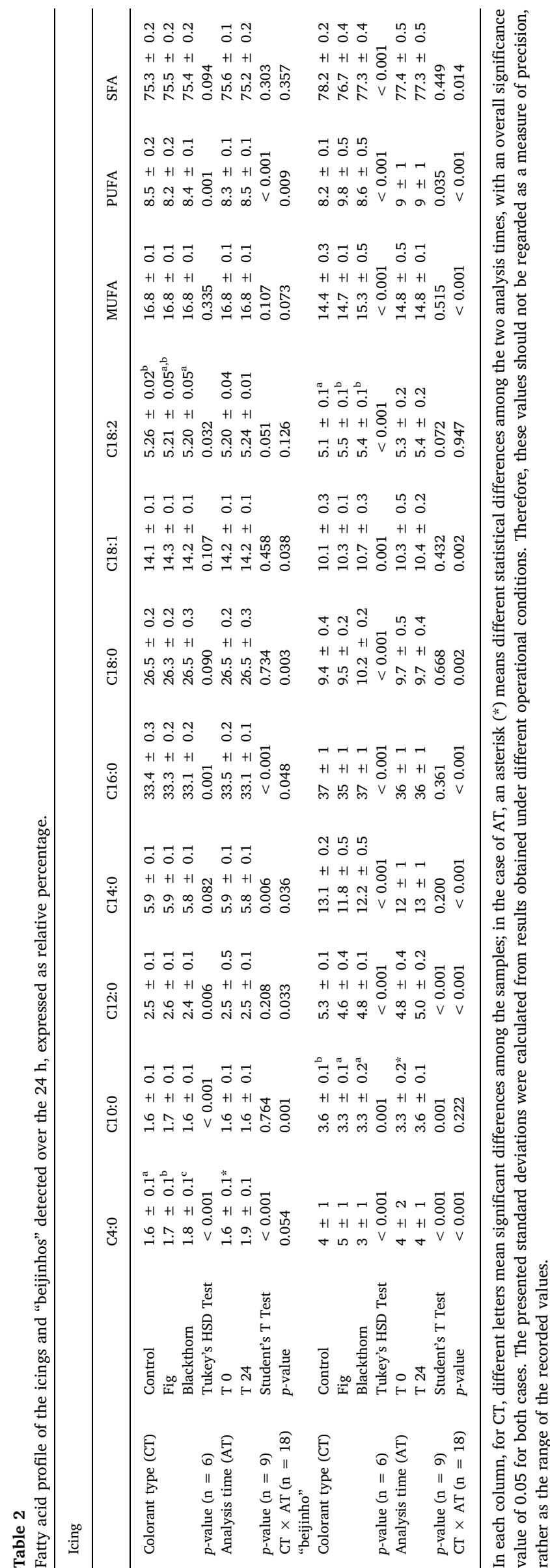

change.

The same four sugars were found in both confectionery products, namely fructose, glucose, sucrose, and lactose, probably due to having very similar ingredients. In both, sucrose was the highest sugar, followed by lactose and then, fructose and glucose in similar amounts. Interestingly, the control sample did not show any fructose or glucose. Sucrose, lactose and total sugars seemed to be more affected by CT than AT, considering the significant differences found among the colored samples. For sucrose, samples with fig extract showed the highest values, followed by those prepared with blackthorn extracts and control samples, all with significant differences. For lactose, CT did not induce differences, but a significantly lower amount was quantified, when compared with the control sample. Finally, for total sugars, significant differences were also found between all three samples, with products prepared with fig extract showing the highest amount, followed by those containing blackthorn extract and control samples. Regarding "beijinhos", the sugar profile showed the same prevalence of sugars as the icing used on doughnuts, with a significant interaction between CT and AT in all cases. Considering the EMM, it was possible to observe (Fig. 1c) that fructose reached the highest values in samples prepared with blackthorn extract, followed by those added with fig extract, and that all samples showed a decrease of this sugar after $24 \mathrm{~h}$. Overall, the changes induced by CT in the nutritional profile were hardly noticeable for both foods, while for the individual sugars, slight changes were found in the icing solution. AT did not induce noticeable differences in these profiles.

\subsection{Fatty acids profile}

Table 2 shows the individual fatty acids (relative percentages) found in both confectionery products. Beyond the tabled fatty acids, 21 other were detected and identified, but in percentages under $1 \%$. The most abundant fatty acid was palmitic acid (C16:0) in both foods, with an average of 30 to $35 \%$, followed by stearic acid (C18:0), which was found in higher quantities in doughnut icing (about 26\%), reaching only $10 \%$ in "beijinhos". The most abundant unsaturated fatty acid was oleic acid (C18:1) with 14\% in the icing and 10\% in "beijinhos". As expected, and due to the inclusion of dairy products, both foods showed higher amounts of saturated fatty acids (SFA), averaging 75\%, followed by monounsaturated (MUFA), at $16 \%$ in the icing solution and $14 \%$ in "beijinhos", and polyunsaturated (PUFA), at roughly $8 \%$ for both cases. Individually, for the icing solution, C4:0 suffered a significant increase from T0 to T24, and all three samples showed significant differences among each other, although slight. The same was found for linoleic acid (C18:2), in which the control sample had a significant difference between blackthorn, but no difference towards the fig extract colored sample. For all other individual fatty acids, a significant interaction was found, as well as for the SFA, MUFA and PUFA, with a very low change in the profile of these molecules. Regarding "beijinhos", a significant interaction was also found in all but two fatty acids, namely C10:0 and C18:2:0. Regarding linoleic acid, it was also detected in higher amounts in samples with the natural colorants, although there was no significant difference from AT. Overall, as intended, the colorants did not significantly change the profile in fatty acids of either foods.

\subsection{Physical analysis}

Table 3 displays the physical analysis performed in the two food samples, which included a $\mathrm{pH}$ determination, external color and texture analysis. Regarding $\mathrm{pH}$, measured with a portable probe, the values did not vary significantly in both foods, ranging from 4.6 to 5.1.

External color analysis was one of the most important analyses, provided that coloring was the main intended effect of the extracts. Thus, the comparison was performed with a portable colorimeter that read the values in three distinct spots of the surface of the foods immediately after production and $24 \mathrm{~h}$ after. The CIELab spherical 


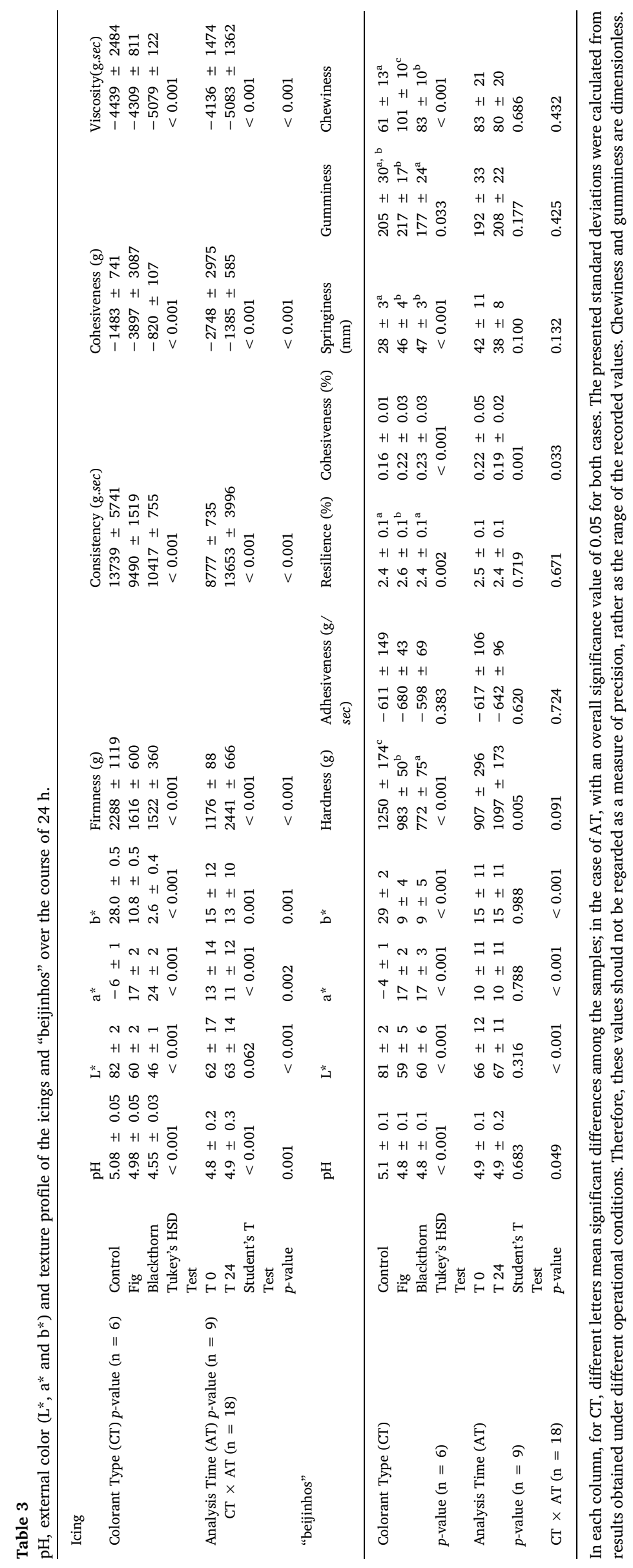




\section{Icing}

\section{Control}

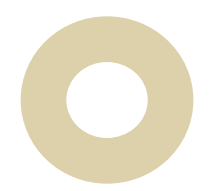

T 24

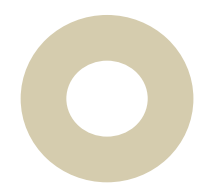

Control

T0
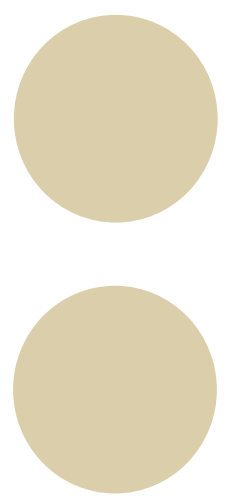

T 24
Fig
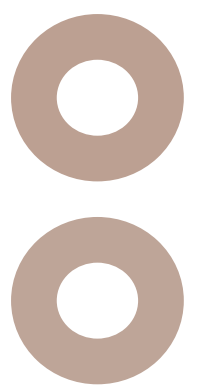

"Beijinhos"

Fig
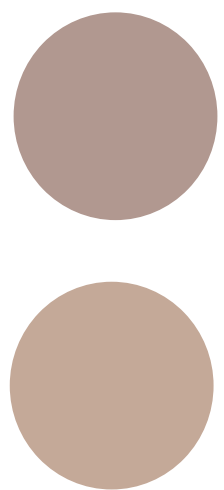

Blackthorn
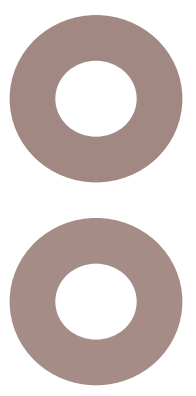

Fig. 2. Detail of variation of colors of the different foods along $24 \mathrm{~h}$, obtained by converting the $\mathrm{L}^{*}, \mathrm{a}^{*}$ and $\mathrm{b}^{*}$ coordinates.

coordinates $\left(\mathrm{L}^{*}, \mathrm{a}^{*}\right.$ and $\left.\mathrm{b}^{*}\right)$ are shown in Table 3 for doughnut icing and "beijinhos". A significant interaction was found for all coordinates, which was expected for CT, but also for AT, which tended to reduce the intensity of the colors for both foods and colorants. As stated above, the statistical treatment implies that in the tables, the different colorant type encompass both analysis times, and each time includes all three samples (control, fig and blackthorn colored). For the specific color assay, the information from the tables can be misleading, and thus, the total difference between the colored samples and the control was calculated using Eq. (4), and the true color (obtained from the conversion of $L^{*}, a^{*}, b^{*}$ coordinates) was calculated and is shown in Fig. 2. This image shows the true colors of the foods, allowing for a better visual interpretation of CT effect. For the doughnut icing, blackthorn rendered a more intense purple color, which lost some of its intensity after $24 \mathrm{~h}$, while the fig colorant did not have such an intense color, despite maintaining it more efficiently. It is also clear that the sample darkened over the 24-hour period. In the case of "beijinhos", both colorants showed very similar coloring capacity, and both lost intensity with AT, becoming lighter. In order to better understand the coloring capacity of these natural colorants, the total difference between the control samples and each colored samples was calculated, for both times, as stated above using Eq. (4). The difference among the fig colored icing and control sample at T0 was 39.3 and after $24 \mathrm{~h}$ reduced to 31.0, while the blackthorn samples started off with a difference from the control sample of 56.4 and reduced to 49.2 , which is consistent with Fig. 2, where a lighter tone is seen for the fig colored sample (lower difference to control sample) and a darker one for blackthorn, and the reduction of color intensity over the $24 \mathrm{~h}$. Regarding "beijinhos", the difference between samples and the control was very similar at T0, namely 42.8 for fig and 44.0 for blackthorn, which reduced considerably for both to 30.1 and 29.3, respectively. The colors, present in Fig. 2, show this very similar behavior between the samples colored with the two different natural extracts, which, compared to the icing colors, shows a harmonization in colors at T0 and T24. This could be due to the different ingredients that induced a loss of color to both samples over the $24 \mathrm{~h}$. The icing color changes were also observed from the EMM plots (Fig. 3), in which it is clear that the darkest color was found in the blackthorn colored samples, which became lighter with time, as with the fig colored samples, while the control sample became darker, as detailed in Fig. 3a, that shows the $L^{*}$ values. The $a^{*}$ coordinates pertain to the green/red variation, and in Fig. $3 \mathrm{~b}$ the higher redness is visible in the colored samples (higher values of $\mathrm{a}^{*}$ ), with blackthorn showing the highest, and a slight reduction over time. Finally, the b* values (Fig. 3c) correspond to the blue/yellow variation, and thus, the colored samples showed the lowest values, closer to the blue tones, while the control sample shifted towards the yellow, with a loss of this tone during the analysis which was not verified for the naturally colored samples.

A texture analysis was also carried out for both foods, although different dimensions were sought for each (the icing solution was a semi-solid, while "beijinhos" are a solid food). The texture analysis is also shown in Table 3. Regarding the icing samples, a significant interaction was found for all dimensions of texture revealing that both AT 

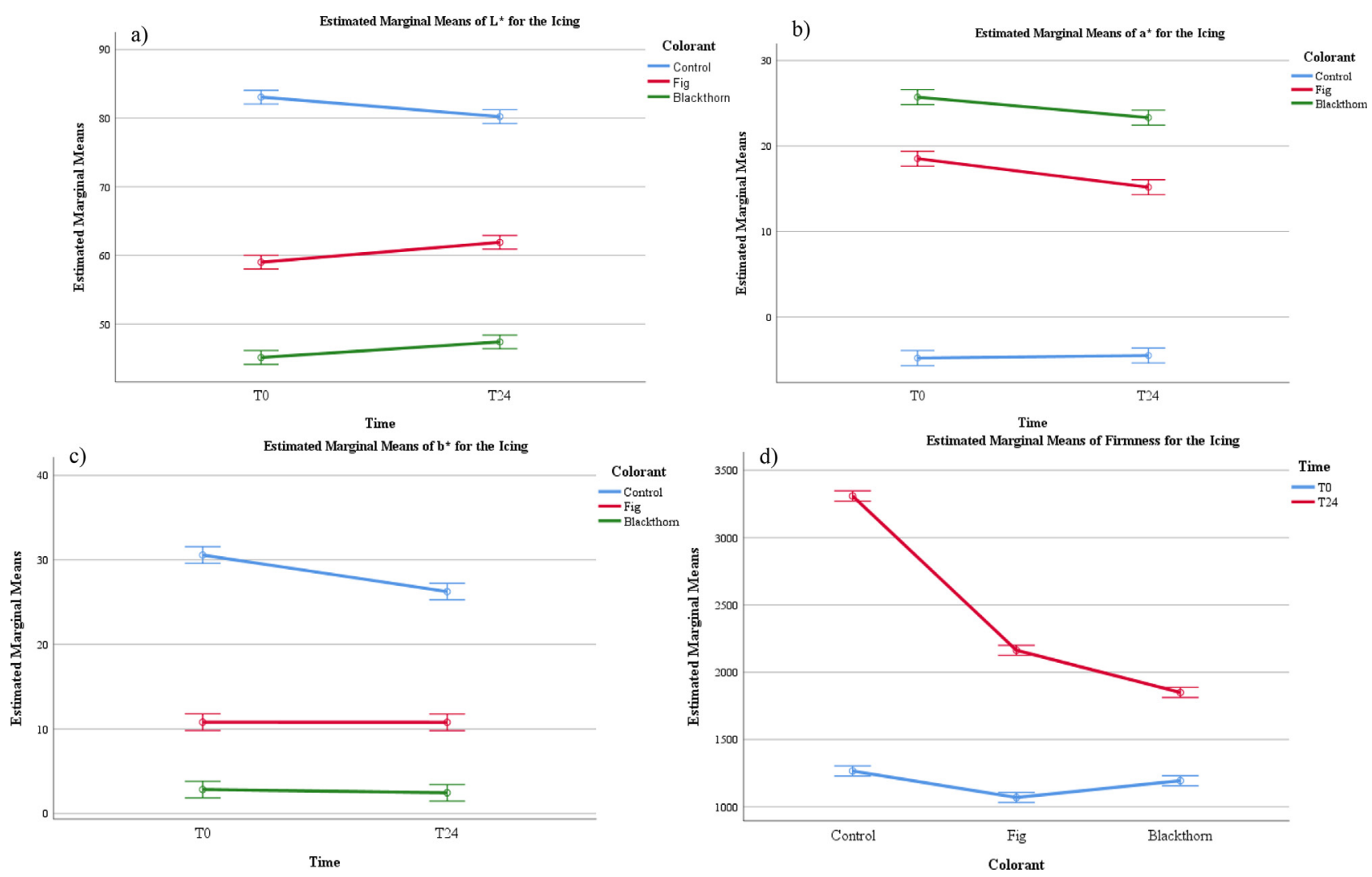

Fig. 3. EMM plots of the icing for a) $L^{*}$, b) $a^{*}$, c) $b^{*}$, and d) Firmness.

and CT contributed to the differences observed in terms of texture. Still, some tendencies were extracted from the EMM plots, namely for firmness (Fig. 3d), in which the plots show that firmness was overall higher for T0; at T24 the control sample showed a much higher firmness value. Firmness is defined as the resistance that a semi-solid food excerpts against deformation of an applied force, and thus, it can be implied that the natural colorants make the icing less firm, making them creamier which is a valued asset in doughnut icing. Another dimension, consistency, can be described as how thick or smooth a substance can be, and is also important for the overall perception of icings. The EMM plot (Fig. S2, supplementary material) shows that at T24 the consistency was higher for all samples, although the control sample showed much higher values, making the icings seem thicker. Overall, in terms of texture profile, the natural colorants seemed to improve the consistency and firmness of the doughnut icing, without having statistically significative influence on the other dimensions, namely cohesiveness and viscosity. The bottom section of Table 3 shows the texture profile of "beijinhos", which, as a solid, underwent a traditional texture profile analysis, having been calculated their hardness, adhesiveness, resilience, cohesiveness, springiness, gumminess and chewiness. Contrarily to the icing texture profile, a significative interaction was only found for cohesiveness, where through the EMM plot, Fig. 1d, it is possible to see that the control sample showed a constant cohesiveness during the $24 \mathrm{~h}$, while the colored samples stared off with a higher cohesiveness and decreased after the $24 \mathrm{~h}$. Cohesiveness is the extent of destruction or deformation a product can endure when a load is applied to it, and thus, the colorants improve this resistance in "beijinhos", making them more resistant to deformation, and to not collapse or deform. Considering hardness (Table 3), the variation along the storage time had a lower influence than the colorants, in which these helped to soften the "beijinhos", with the softer one being the blackthorn colored "beijinho" followed by fig colored, with statistical differences among each other and towards the control sample. Adhesiveness did not show a significant interaction, but there were no significant differences among AT and CT. Resilience showed similar behavior to hardness, without significant changes induced by AT, contrarily to CT, namely the fig coloring, which induced higher resilience with significative differences to the control and blackthorn colored samples. Springiness, gumminess and chewiness did not have significative changes induced by the AT, but CT did induce some statistically significant changes. The colorants induced a significant increase in springiness and, therefore a higher chewiness, while gumminess increased more drastically in the fig colored sample. Overall, the colorants showed a higher impact over "beijinhos" than over the icings, probably due to the specific method of making them into a round shape. Overall, the colorants, beyond the coloring effect, increased the softness and made them chewier, which are favored attributes for these candies.

\section{Conclusion}

The main objective of this work was to uncover the effects that two natural colorants (fig peel and blackthorn fruit) could have in two different food uses, doughnut icings and "beijinhos", two appreciated dairy snacks. Overall, the main objective of maintaining an acceptable coloring effect for at least $24 \mathrm{~h}$ without changing nutritional properties (in line with food additive regulations) was achieved. For doughnut icing, blackthorn conferred a darker purple color, while fig only render a light pink color. In turn, while blackthorn lost a considerable tone after $24 \mathrm{~h}$, the fig maintained its color reasonably unaltered. Inversely, both colorants showed a pleasing dark purple color at the beginning of the experiment for "beijinhos", and both tended to lose this tone and become closer to pink. This change in the first color could be due to some ingredient of the icing which quickly oxidized the fig compounds, thus promoting a loss in color intensity. In terms of texture, the icings profited in terms of lower firmness and consistency offered by the colorants, although these were just general tendencies. The "beijinhos", on the other hand, showed significant changes induced by CT, namely on hardness reduction and increase in springiness and chewiness. The 
chemical profile of these foods only underwent very slight changes, with moisture reduction in the icings, and a slight increase in soluble sugars (fatty acids were kept nearly unaltered). Likewise, "beijinhos" were also very slightly altered, with a small increase in moisture and carbohydrates and no considerable change in fatty acids. Overall the colorants improved the texture properties, while conferring color with no apparent change in the chemical profile. Still, during the $24 \mathrm{~h}$, no statistical changes occurred in the studied parameters, and both foods could profit from an extension of the shelf-life (although no legal shelflife is advised from competent authorities). More studies in terms of oxidation and bacterial growth are needed to further corroborate this claim. Fig peel and blackthorn are interesting colorants that could be considered as food colorants due to their natural origin which might be consumed without posing toxicological risks towards consumers.

\section{CRediT authorship contribution statement}

Emanueli Backes: Investigation. Maria G. Leichtweis: Investigation. Carla Pereira: Investigation, Conceptualization. Marcio Carocho: Data curation, Writing - original draft. Joảo C.M. Barreira: Data curation, Writing - review \& editing. Aziza Kamal Genena: Writing - review \& editing. Ilton José Baraldi: Writing - review \& editing, Conceptualization. Maria Filomena Barreiro: Writing - review \& editing. Lillian Barros: Investigation, Conceptualization, Writing review \& editing. Isabel C.F.R. Ferreira: Project administration, Conceptualization, Writing - review \& editing.

\section{Declaration of Competing Interest}

The authors declare that they have no known competing financial interests or personal relationships that could have appeared to influence the work reported in this paper.

\section{Acknowledgments}

The authors would like to thank the Foundation for Science and Technology (FCT, Portugal) and FEDER, under Program PT2020 for financing CIMO (Centro de Investigação de Montanha) (UID/AGR/ 00690/2019). This work was further funded by the European Structural and Investment Funds (FEEI) through the Regional Operational Program North 2020 within the scope of the Mobilizador ValorNatural ${ }^{\circledast}$ project, to whom the author M. Carocho thanks for his contract. Further acknowledgments are due to the national funding by FCT, P.I., through the institutional scientific employment program-contract for L. Barros' contracts, and though the celebration of program-contract foreseen in No. 4, 5 and 6 of article $23^{\circ}$ of Decree-Law No. 57/2016, of 29th August, amended by Law No. 57/2017, of 19th July for C. Pereira's contract.

\section{Appendix A. Supplementary data}

Supplementary data to this article can be found online at https:// doi.org/10.1016/j.foodchem.2020.127457.

\section{References}

Agcam, E., Akyildiz, A., \& Balasubramaniam, V. M. (2017). Optimization of anthocyanins extraction from black carrot pomace with thermosonication. Food Chemistry, 237, 461-470.

Amessis-Ouchemoukh, N., Ouchemoukh, S., Meziant, N., Idiri, Y., Hernanz, D., Stinco, C. M., ... Luis, J. (2017). Bioactive metabolites involved in the antioxidant, anticancer and nticalpain activities of Ficus carica L., Ceratonia siliqua L. and Quercus ilex L. extracts. Industrial Crops and Products, 95, 6-17.

AOAC. (2016). Official Methods of Analysis of AOAC International. In AOAC (Association of Official Analytical Chemists) International. Arlington.

Backes, E., Pereira, C., Barros, L., Prieto, M. A., Genena, A. K., Barreiro, M. F., \& Ferreira, I. C. F. R. (2018). Recovery of bioactive anthocyanin pigments from Ficus carica L. peel by heat, microwave, and ultrasound based extraction techniques. Food Research
International, 113, 197-209.

Barolo, M. I., Ruiz Mostacero, N., \& López, S. N. (2014). Ficus carica L. (Moraceae): An ancient source of food and health. Food Chemistry, 164, 119-127.

Barros, L., Carvalho, A. M., Morais, J. S., \& Ferreira, I. C. F. R. (2010). Strawberry-tree, blackthorn and rose fruits: Detailed characterisation in nutrients and phytochemicals with antioxidant properties. Food Chemistry, 120(1), 247-254.

Barros, L., Pereira, E., Calhelha, R. C., Dueñas, M., Carvalho, A. M., Santos-Buelga, C., \& Ferreira, I. C. F. R. (2013). Bioactivity and chemical characterization in hydrophilic and lipophilic compounds of Chenopodium ambrosioides L. Journal of Functional Foods, $5,1732-1740$.

Buenrostro-Figueroa, J. J., Velázquez, M., Flores-Ortega, O., Ascacio-Valdés, J. A., Huerta-Ochoa, S., Aguilar, C. N., \& Prado-Barragán, L. A. (2017). Solid state fermentation of fig (Ficus carica L.) by-products using fungi to obtain phenolic compounds with antioxidant activity and qualitative evaluation of phenolics obtained. Process Biochemistry, 62(186), 16-23.

Céspedes, C. L., El-Hafidi, M., Pavon, N., \& Alarcon, J. (2008). Antioxidant and cardioprotective activities of phenolic extracts from fruits of Chilean blackberry Aristotelia chilensis (Elaeocarpaceae), Maqui. Food Chemistry, 107(2), 820-829.

CLSI Document M100-S18. Performance Standards for Antimicrobial Susceptibility Testing. Clinical and Laboratory Standards Institute 2008 Wayne, PA, USA.

Diao, W. R., Hu, Q. P., Feng, S. S., Li, W. Q., \& Xu, J. G. (2013). Chemical composition and antibacterial activity of the essential oil from green huajiao (Zanthoxylum schinifolium) against selected foodborne pathogens. Journal of Agricultural and Food Chemistry, 61(25), 6044-6049.

Duenas, M., Perez-Alonso, J. J., Santos-Buelga, C., \& Escribano-Bailon, T. (2008). Anthocyanin composition in fig (Ficus carica L.). Journal of Food Composition and Analysis, 21(2), 107-115.

EUCAST (2013). European Committee on Antimicrobial Susceptibility Testing (EUCAST). Stockholm, Sweden: European Society of Clinical Microbiology and Infectious Diseases (ESCMID).

European Parliament. (2011). Regulation (UE) no 1129/2011 da Comissão Europeia. In Jornal Oficial da União Europeia.

Gordillo, B., Sigurdson, G. T., Lao, F., González-Miret, M. L., Heredia, F. J., \& Giusti, M. M (2018). Assessment of the color modulation and stability of naturally copigmented anthocyanin-grape colorants with different levels of purification. Food Research International, 106, 791-799.

Guimarães, R., Barros, L., Calhelha, R. C., Carvalho, A. M., Queiroz, M. J. R. P., \& Ferreira, I. C. F. R. (2014). Bioactivity of different enriched phenolic extracts of wild fruits from northeastern Portugal: A comparative study. Plant Foods for Human Nutrition, 69(1), 37-42.

Guimarães, R., Barros, L., Dueñas, M., Carvalho, A. M., Queiroz, M. J. R. P., SantosBuelga, C., \& Ferreira, I. C. F. R. (2013). Characterisation of phenolic compounds in wild fruits from Northeastern Portugal. Food Chemistry, 141(4), 3721-3730.

Harzallah, A., Bhouri, A. M., Amri, Z., Soltana, H., \& Hammami, M. (2016). Phytochemical content and antioxidant activity of different fruit parts juices of three figs (Ficus carica L.) varieties grown in Tunisia. Industrial Crops and Products, 83, 255-267.

Khaliq, A., Ahmad, S. D., Sabir, S. M., \& Khan, A. (2015). Antioxidant activity and inhibitory effect of cultivars of Olive (Olea europaea) against lipid peroxidation in mice liver. Turkish Journal of Biochemistry, 40(2), 188-196.

Kuete, V., Ango, P. Y., Fotso, G. W., Kapche, G. D. W. F., Dzoyem, J. P., Wouking, A. G., .. Abegaz, B. M. (2011). Antimicrobial activities of the methanol extract and compounds from Artocarpus communis (Moraceae). BMC Complementary and Alternative Medicine, 25, 11-42.

Leichtweis, M. G., Pereira, C., Prieto, M. A., Barreiro, M. F., Baraldi, I. J., Barros, L., \& Ferreira, I. C. F. R. (2019). Ultrasound as a rapid and low-cost extraction procedure to obtain anthoxyanin-based colorants from Prunus spinosa L. fruit epicarp: Comparative study with conventional heat-based extraction. Molecules, 24.

Liu, Z., Pan, Y., Li, X., Jie, J., \& Zeng, M. (2017). Chemical composition, antimicrobial and anti-quorum sensing activities of pummelo peel flavonoid extract. Industrial Crops and Products, 109, 862-868.

Mojica, L., Berhow, M., \& Gonzalez de Mejia, E. (2017). Black bean anthocyanin-rich extracts as food colorants: Physicochemical stability and antidiabetes potential. Food Chemistry, 229, 628-639.

Morales, P., Ferreira, I. C. F. R., Carvalho, A. M., Fernández-Ruiz, V., Sánchez-Mata, M. S. O. S. C. C., Cámara, M., ... Tardío, J. (2013). Wild edible fruits as a potential source of phytochemicals with capacity to inhibit lipid peroxidation. European Journal of Lipid Science and Technology, 115(2), 176-185.

Oliveira, A. P., Valentão, P., Pereira, J. A., Silva, B. M., Tavares, F., \& Andrade, P. B. (2009). Ficus carica L.: Metabolic and biological screening. Food and Chemical Toxicology, 47(11), 2841-2846.

Pereira, C., López-Corrales, M., Serradilla, M. J., Villalobos, M. del C., Ruiz-Moyano, S., \& Martín, A. (2017). Influence of ripening stage on bioactive compounds and antioxidant activity in nine fig (Ficus carica L.) varieties grown in Extremadura, Spain. Journal of Food Composition and Analysis, 64, 203-212.

Pertuzatti, P. B., Barcia, M. T., Rebello, L. P. G., Gómez-Alonso, S., Duarte, R. M. T. Duarte, M. C. T., ... Hermosín-Gutiérrez, I. (2016). Antimicrobial activity and differentiation of anthocyanin profiles of rabbiteye and highbush blueberries using HPLC-DAD-ESI-MSn and multivariate analysis. Journal of Functional Foods, 26, $506-516$.

Pinacho, R., Cavero, R. Y., Astiasarán, I., Ansorena, D., \& Calvo, M. I. (2015). Phenolic compounds of blackthorn (Prunus spinosa L.) and influence of in vitro digestion on their antioxidant capacity. Journal of Functional Foods, 19, 49-62.

Solomon, A., Golubowicz, S., Yablowicz, Z., Grossman, S., Bergman, M., Gottlieb, H. E., Flaishman, M. A. (2006). Antioxidant activities and anthocyanin content of fresh fruits of common fig (Ficus carica L.). Journal of Agricultural and Food Chemistry, 
54(20), 7717-7723.

Takebayashi, J., Iwahashi, N., Ishimi, Y., \& Tai, A. (2012). Development of a simple 96well plate method for evaluation of antioxidant activity based on the oxidative haemolysis inhibition assay (OxHLIA). Food Chemistry, 134(1), 606-610.

Uz, R., Bakar, N. H. A., Swethadri, G. K., Baig, A., Idria, M. A., \& Maryam, I. U. (2015). Non-toxic antiproliferative effect of Ficus carica fruit extracts on estrogen receptor positive breast cancer cell (MCF-7). Journal of Chemical and Pharmaceutical Research,
$7(10), 815-821$.

Vallejo, F., Marín, J. G., \& Tomás-Barberán, F. A. (2012). Phenolic compound content of fresh and dried figs (Ficus carica L.). Food Chemistry, 130(3), 485-492.

Yao, W. R., Wang, H. Y., Wang, S. T., Sun, S. L., Zhou, J., \& Luan, Y. Y. (2011). Assessment of the antibacterial activity and the antidiarrheal function of flavonoids from bayberry fruit. Journal of Agricultural and Food Chemistry, 59, 5312-5317. 